University of Nebraska - Lincoln

DigitalCommons@University of Nebraska - Lincoln

2009

\title{
Asymmetric synthesis of 1,2-dioxanes: Approaches to the peroxyplakoric acids
}

\author{
Chunping $\mathrm{Xu}$ \\ University of Nebraska-Lincoln \\ Chris Schwartz \\ University of Nebraska-Lincoln \\ Joseph Raible \\ University of Nebraska-Lincoln \\ Patrick Dussault \\ University of Nebraska-Lincoln, pdussault1@unl.edu
}

Follow this and additional works at: https://digitalcommons.unl.edu/chemistrydussault

Part of the Chemistry Commons

Xu, Chunping; Schwartz, Chris; Raible, Joseph; and Dussault, Patrick, "Asymmetric synthesis of 1,2-dioxanes: Approaches to the peroxyplakoric acids" (2009). Patrick Dussault Publications. 9. https://digitalcommons.unl.edu/chemistrydussault/9

This Article is brought to you for free and open access by the Published Research - Department of Chemistry at DigitalCommons@University of Nebraska - Lincoln. It has been accepted for inclusion in Patrick Dussault Publications by an authorized administrator of DigitalCommons@University of Nebraska - Lincoln. 


\title{
Asymmetric synthesis of 1,2-dioxanes: Approaches to the peroxyplakoric acids
}

\author{
Chunping Xu, Chris Schwartz, Joseph Raible and Patrick H. Dussault \\ Department of Chemistry, University of Nebraska-Lincoln, Lincoln, NE 68588-0304 \\ Corresponding author - P. H. Dussault, tel 402 472-6951, fax 402 472-9402, email pdussault1@unl.edu
}

\begin{abstract}
The stereospecific intramolecular alkylation of a hydroperoxyacetal provides the basis for the first asymmetric synthesis of the dioxane propionate core of the peroxyplakorates. Chemoselective hydrometallation of an alkyne in the presence of a peroxide is used to introduce a synthon for the polyunsaturated side chains of the peroxyplakorates. The route suggests a general solution for the 1,2-dioxane unit in many peroxide natural products.
\end{abstract}

Keywords: peroxyplakoric, 1,2-dioxane, peroxide, intramolecular alkylation

\section{Introduction}

The peroxyplakoric acids are a family of marine natural products sharing a 6-methoxy-1,2-dioxane-3-propionic acid core, ${ }^{1,}$ 2 and differing in the stereochemistry at $\mathrm{C}_{3}$ and in the geometry and substitution of the diene within the $\mathrm{C}_{6}$ tail (Figure 1). Several members of the family, as well as some simplified analogs, possess significant antifungal or antimalarial activity. ${ }^{3-4}$ However, there has been no reported synthesis of a peroxyplakoric acid, ${ }^{5}$ nor has there been a general strategy for asymmetric synthesis of alkoxy-1,2-dioxanes. ${ }^{6}$ We now report an asymmetric synthesis of 6-alkoxy-3,6-dialkyl-1,2-dioxanes by a route that should be broadly applicable to 1,2-dioxanes, one of the most common substructures in peroxide natural products. $1,7,8$

\section{Background}

The two major challenges in the asymmetric synthesis of the peroxyplakorates are stereoselective introduction of the 3-alkoxy-3,6-dialkyl substituted 1,2-dioxane, and incorporation of both an oxidation-sensitive side chain and a reductionsensitive 1,2-dioxane.

Most reported methods for the synthesis of 1,2-dioxanes involve electrophilic or radical cyclizations ${ }^{9-11}$ and are, in general, poorly suited for an approach to the peroxyplakorates. However, the 1,2-dioxane propionate core can be formed through conjugate addition of a hydroperoxy enoate. This reaction, first observed by Bartlett and co-workers during investigations of intramolecular nucleophilic epoxidations, ${ }^{12}$ was subsequently optimized for the synthesis of alkoxydioxanes, ${ }^{3}$ and has been applied by several groups to the synthesis of simplified peroxyplakorate analogs. ${ }^{4}$ However, a conjugate addition offers no obvious means of controlling the relative stereochemistry of the side chain and dioxane stereocenters. ${ }^{13}$ Furthermore, in the course of a recent total synthesis of structurally simpler peroxyacarnoates, ${ }^{8,14}$ we found that conjugate addition predominantly furnished the 1,2-dioxane isomer with $\mathrm{C}_{3}$ and $\mathrm{C}_{6}$ alkyl substituents in a trans (diequatorial) relationship; in the case of the peroxyplakorates, this would preclude any approach to the B-series of diastereomers. Finally, at the time these studies were initiated, conjugate addition offered no means for control of absolute stereochemistry; a recent report on organocatalyst-mediated 5-exo-trig cyclizations of simple hydroperoxides offers some hope in this regard. ${ }^{15}$

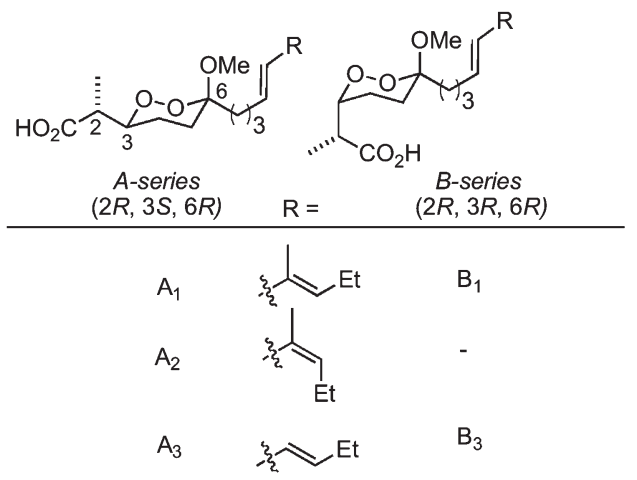

Figure 1. Peroxyplakoric acids. 
The use of a conjugate addition also requires introduction of a hydroperoxyacetal nucleophile in the presence of an enoate (Scheme 1). We found a reported acid-catalyzed peroxyacetalization $^{3 a}$ to be very substrate dependent, something that has also been noted by others. ${ }^{4}$ Model studies suggested that selective ozonolysis of a $\mathrm{C}_{6}$-methylene, a reaction we had successfully employed in the presence of a disubstituted enoate (Scheme 1, $\mathrm{X}=\mathrm{H}),{ }^{14}$ was problematic in the presence of the trisubstituted enoate required for the peroxyplakorates (Scheme $1, \mathrm{X}=\mathrm{CH}_{3}$ ).

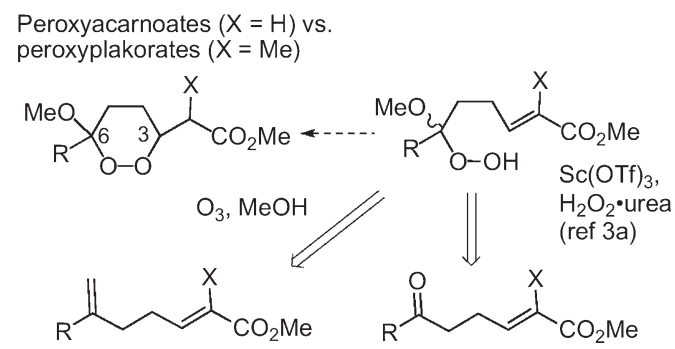

Scheme 1. Methods for introduction of the hydroperoxyacetal.

We became interested in a general approach to asymmetric synthesis of dioxanes based upon intramolecular nucleophilic substitution. Cyclization of a hydroperoxyepoxide has been applied to the asymmetric synthesis of the antimalarial Yingzhaosu A. ${ }^{16}$ However, the homologous opening of secondary oxetanes proved unsuccessful. ${ }^{17}$ These limitations led us to pursue intramolecular alkylations of hydroperoxyketals. We had previously observed the efficiency of intramolecular hydroperoxide alkylations in a 6-exo tet displacement of a primary iodide ${ }^{18}$; more recently, we employed a corresponding displacement of a secondary methanesulfonate as part of the synthesis of spiro bisperoxyacetals (Scheme 2). ${ }^{19}$

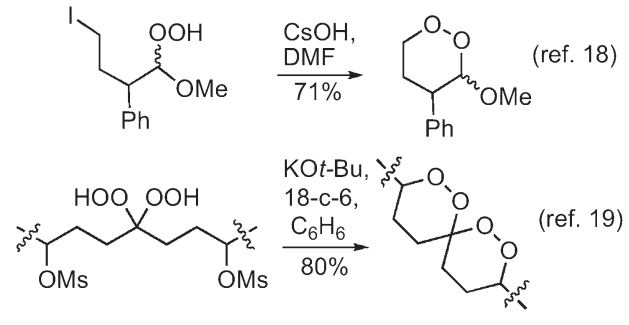

Scheme 2. Intramolecular displacements with hydroperoxyacetals.

Our retrosynthetic approach (Scheme 3), illustrated for peroxyplakoric acid $\mathrm{A}_{3}$, is designed to allow a general solution to any member of the family. As the diene side chains in the peroxyplakorates are incompatible with ozonolytic introduction of the hydroperoxyacetal, we planned to employ Pd-mediated $\mathrm{sp}^{2} / \mathrm{sp}^{2}$ couplings as a disconnect to a metalloalkene or iodoalkene. ${ }^{20}$ What remained to be seen was whether the functionalized alkene could be installed via hydrometallation of an alkynyl-substituted dioxane. The 1,2-dioxane ring would be installed through a stereospecific intramolecular displacement of a secondary sulfonate by a hydroperoxyacetal, which would in turn be revealed through ozonolysis of an alkene. Our previous work with the peroxyacarnoates had suggested that the terminal alkyne would be stable toward ozonolytic introduction of the hydroperoxyketal. ${ }^{8,14}$ The secondary alcohol precursor of the sulfonate leaving group would be introduced via an asymmetric aldol reaction, with the choice of syn- or antiselective aldol determining the choice of peroxyplakoric A or B-series.

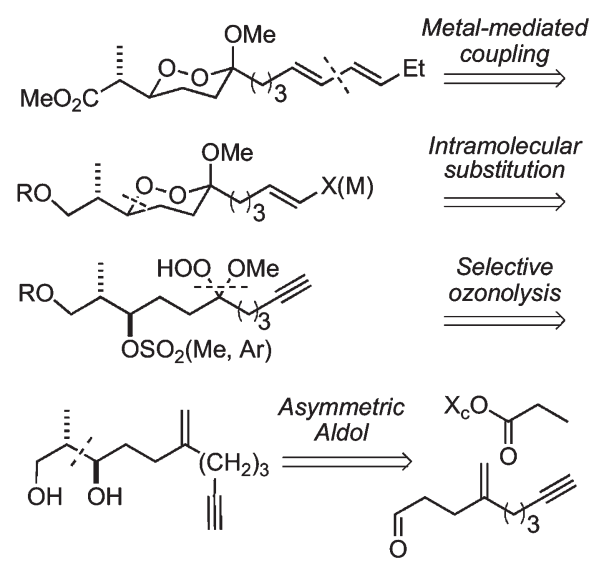

Scheme 3. Retrosynthesis of Peroxyplakorate $\mathrm{A}_{3}$.

Synthesis of the functionalized 1,2-dioxane core is illustrated in Scheme 4. As our initial target, we chose the peroxyplakorate 'A' series. The 2,3-syn stereochemistry requires an anti-aldol precursor. The dialkylboronate derived from chiral ester $\mathbf{1}^{21}$ underwent reaction with aldehyde $2^{14}$ to furnish a good yield of a mixture of aldol products in which the desired $(2 R, 3 R)$-anti diastereomer (3) predominated; the only significant byproduct $(8 \%)$ was the syn-2S,3R-diasteromer. Reduction of 3 to the 1,3-diol 4 was followed by selective protection of the primary alcohol to form the triisopropylsilyl ether (5). Although attempted activation of the $\mathrm{C}_{3}$-hydroxyl as the triflate resulted in significant decomposition, the corresponding mesylate 6 was easily prepared. Methanolic ozonolysis of the methylene group furnished a high yield of a 1:1 mixture of hydroperoxyacetals $\mathbf{7 a}$ and $\mathbf{7 b}$, which were incompletely separated even by semi-preparative HPLC and therefore carried on as a mixture. ${ }^{22}$ Treatment with potassium tert-butoxide in the presence of 18-crown-6 furnished a 1:1 mixture of diastereomeric 1,2-dioxanes $\mathbf{8 a}$ and $\mathbf{8 b}$ in excellent yield. The configurational assignment of dioxane 8a was based upon a combination of precedent for the aldol reaction, as well as the ${ }^{3} J_{\mathrm{H}}$ constants for $\mathrm{H}_{3}(11.0,6.3,1.9 \mathrm{~Hz})$ in the ${ }^{1} \mathrm{H}$ NMR spectrum,
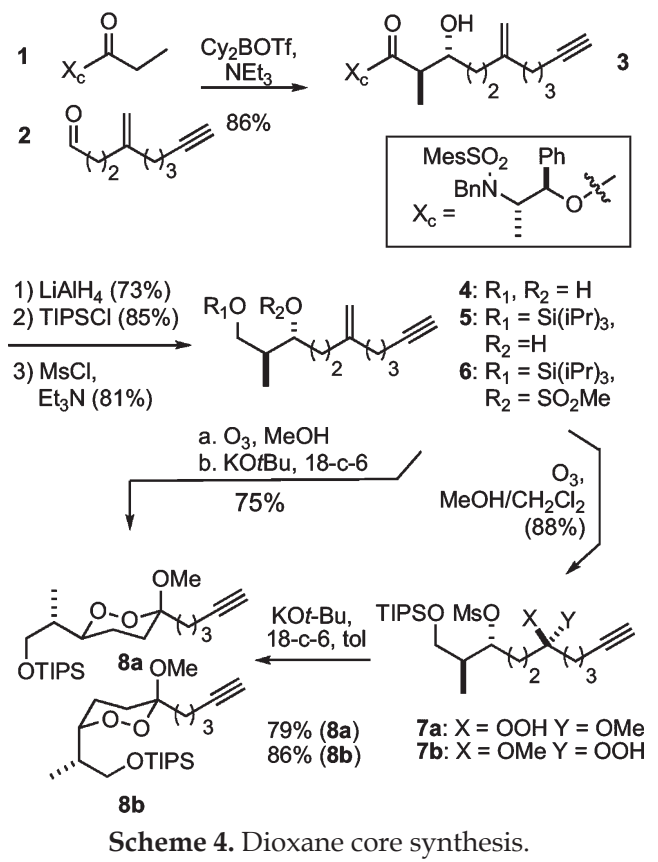
which were very similar to the values reported for peroxyplakoric acids $\mathrm{A}_{1}-\mathrm{A}_{3}$ and consistent with typical observations for 1,2-dioxane natural products bearing an axial hydrogen at $\mathrm{C}_{3}{ }^{2}{ }^{2}{ }^{23}$ Interestingly, the undesired peroxyacetal $\mathbf{8 b}$ failed to epimerize in the presence of mild acid and was decomposed by strong acid (e.g., methanolic $\mathrm{HCl}$ ).

Small quantities of the individual diastereomers $7 \mathbf{a}$ and $7 \mathbf{b}$ could be obtained by analytical HPLC. Subjecting the individual diastereomers to the cyclization conditions furnished the diastereomerically pure 1,2-dioxanes $\mathbf{8 a}$ and $\mathbf{8 b}$, respectively (Scheme 4), demonstrating that the cyclization was in fact stereospecific and allowing stereochemical assignment of the hydroperoxyacetals.

Chemoselective functionalization of the alkynyl dioxane to an E-iodoalkenyl dioxane was readily achieved by hydrozirconation with the Schwartz reagent, followed by quenching with iodine (Scheme 5). Our earlier studies had demonstrated the compatibility of dialkyl peroxides with a number of Pd-mediated $\mathrm{sp}^{2} / \mathrm{sp}^{2}$ couplings. ${ }^{14,}$ 20, 24 Cross coupling of the alkenyl iodide 9 with an $E$-butenyl zinc reagent derived from hydrozirconation of 1-butyne furnished a nearly inseparable 1:3 mixture of the desired diene and the terminal alkene derived from a net reduction. ${ }^{25} \mathrm{~A}$ small amount of diene, separated through careful chromatography, was treated with fluoride to reveal 12, the primary alcohol precursor of the peroxyplakorate $A_{3}$.
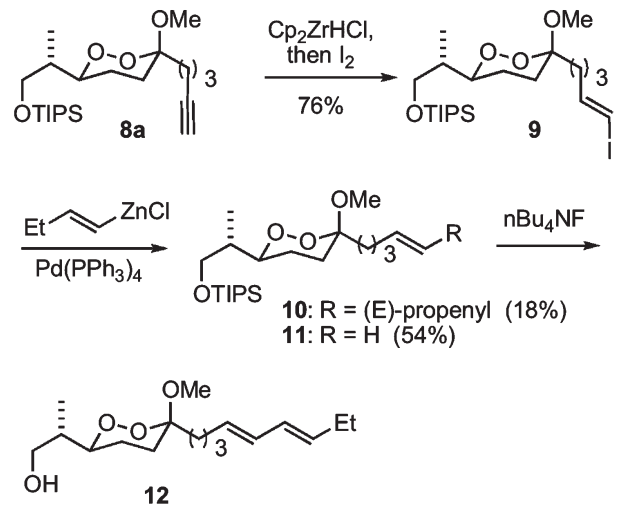

Scheme 5. Negishi coupling.

\section{Conclusion}

We have demonstrated an approach to the peroxyplakoric acid family of 1,2-dioxanes by a route, which, through choice of aldol precursor or the organometallic coupling partner, should be applicable to any member of the peroxyplakorates. The stereospecific intramolecular alkylation at the heart of the approach suggests a new and broadly applicable asymmetric approach to 1,2-dioxane natural products, which include a secondary peroxide linkage ${ }^{1,6}$; two examples are shown below (Figure 2). ${ }^{26-27}$

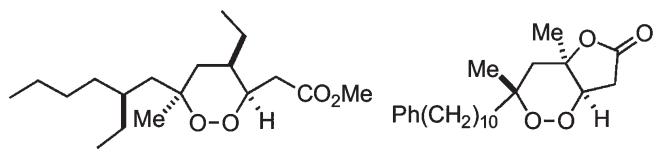

Plakortin (ref. 26) Plakortolide E (ref. 27)

Figure 2. 1,2-Dioxanes with secondary peroxide elements.

\section{Experimental}

\subsection{General procedures}

Dichloromethane and THF were distilled from $\mathrm{CaH}_{2}$ and $\mathrm{Na}$ /benzophenone, respectively. All other reagents were used as received, except as noted. All reactions were conducted under an atmosphere of nitrogen except where noted. Thin layer chromatography (TLC) was performed on $0.25 \mathrm{~mm}$ hard-layer silica G plates; developed plates were visualized with UV lamp and/or by staining: $1 \%$ Ceric sulfate and 10\% ammonium molybdate in $10 \% \mathrm{H}_{2} \mathrm{SO}_{4}$ (general stain, after charring); $1 \% \quad N, N^{\prime}$-dimethyl-p-phenylenediamine solution in 1:20:100 acetic acid/water/methanol (specific for peroxides) $28 ; 1 \%$ aq $\mathrm{KMnO}_{4}$ (for unsaturated compounds). "Standard drying and purification (solvent)" refers to drying of organic extracts over $\mathrm{Na}_{2} \mathrm{SO}_{4}$, removal of solvent under vacuum, and purification by flash chromatography using the indicated eluting solvent. Analytical HPLC was performed on a $4.6 \mathrm{~mm} \times 25 \mathrm{~cm}$ Si column $(5 \mu \mathrm{m})$; semi-preparative HPLC on a $21.4 \mathrm{~mm} \times 25 \mathrm{~cm}$ Si column $(8 \mu \mathrm{m})$; both employed RI detection. ${ }^{1} \mathrm{H}$ and ${ }^{13} \mathrm{C}$ spectra were recorded at 300,400 , or $500 \mathrm{MHz}$ in $\mathrm{CDCl}_{3}$ unless otherwise indicated; peaks are reported as: chemical shift (number of protons, multiplicity, $J$ couplings in $\mathrm{Hz}$ ). Infrared spectra were recorded as neat ATR films with selected absorbances reported in wavenumbers $\left(\mathrm{cm}^{-1}\right)$. Optical rotations were measured on a digital polarimeter at ambient temperature.

\section{2. (1R,2S)-2-(N-Benzyl-N-mesitylenesulfonyl)amino-1-phe- nyl-1-propyl, propionate ester (1)}

Compound 1 was prepared in three steps from norephedrine by a slight variant of the reported procedure in which the crude propionate 1 was not triturated with hexane but directly recrystallized from EA/Hex. ${ }^{21}$

\subsection{4-Methylenenon-8-ynal (2)}

Compound 2 was prepared in three steps from 5-hexynoic acid by the method described in $\mathrm{Xu}$ et al. ${ }^{14}$ The volatile and sensitive aldehyde was used without purification; ${ }^{1} \mathrm{H}$ NMR $\left(\mathrm{CDCl}_{3}\right) \delta 9.6(1 \mathrm{H}, \mathrm{t}, \mathrm{J}=1.6 \mathrm{~Hz}), 4.6-4.5(2 \mathrm{H}, \mathrm{m}), 2.39(2 \mathrm{H}$, $\mathrm{dt}, J=1.6,7.6 \mathrm{~Hz}), 2.1(2 \mathrm{H}$, br t, $J=7.5 \mathrm{~Hz}), 1.99(2 \mathrm{H}, \mathrm{dt}, J=2.6$, $7.1 \mathrm{~Hz}), 1.94(2 \mathrm{H}$, br t, J=7.9 Hz), $1.8(1 \mathrm{H}, \mathrm{t}, J=2.6 \mathrm{~Hz}), 1.5-1.4$ $(2 \mathrm{H}, \mathrm{m}$ but overall quintet $J \sim 7.4 \mathrm{~Hz}) ;{ }^{13} \mathrm{C} \mathrm{NMR}\left(\mathrm{CDCl}_{3}\right) \delta$ 201.9, 146.6, 110.1, 84.0, 68.7, 41.7, 35.0, 27.9, 26.3, 17.8.

\subsection{Dicyclohexyl(trifluoromethylsulfonyloxy)borane}

Dicyclohexylboron triflate was prepared by a slight modification of the method of Inoue et al., ${ }^{21}$ in which the biphase obtained by allowing the crude reaction mixture to stand was separated by withdrawal of the lower phase with a syringe and needle.

\section{5. (2R,3R)[(1R,2S)-2-(N-Benzyl-2,4,6-trimethylphenyl- sulfonamido)-1-phenylpropyl]3-hydroxy-2-methyl-6- methyleneundec-10-ynoate (3)}

Compound 3 was prepared from the chiral propionate 1 and aldehyde 2 by a variant of the method of Inoue et al. ${ }^{21}$ Into an oven-dried $250 \mathrm{~mL}$ round bottom flask under nitrogen was added $(1 R, 2 S)-2-(N$-benzyl- $N$-mesitylenesulfonyl)amino1-phenyl-1-propyl propionate $(3.1 \mathrm{~g}, 6.5 \mathrm{mmol})$ and $40 \mathrm{~mL}$ $\mathrm{CH}_{2} \mathrm{Cl}_{2}$. To this solution was added $\mathrm{Et}_{3} \mathrm{~N}(2.2 \mathrm{~mL}, 16 \mathrm{mmol})$ via syringe. The solution was cooled to $-78^{\circ} \mathrm{C}$ and a solution of dicyclohexylboron triflate $(1.0 \mathrm{M}$ in hexane, $13 \mathrm{~mL}, 13 \mathrm{mmol}$ ) was added dropwise over $15 \mathrm{~min}$. The resulting solution was stirred at $-78^{\circ} \mathrm{C}$ for $2 \mathrm{~h}$. Crude 4-methylenenon-8-ynal $(1.2 \mathrm{~g}$, $7.9 \mathrm{mmol}$ ) in $5 \mathrm{~mL} \mathrm{CH} \mathrm{Cl}_{2}$ was added dropwise to the enol boronate solution. The reaction mixture was stirred for $1 \mathrm{~h}$ at $-78{ }^{\circ} \mathrm{C}$ and was allowed to warm to room temperature over $1 \mathrm{~h}$, then quenched by addition of $0.1 \mathrm{M} \mathrm{pH} 7$ phosphate buffer 
solution $(24 \mathrm{~mL})$. The mixture was diluted with $120 \mathrm{~mL}$ methanol, after which, $30 \% \mathrm{H}_{2} \mathrm{O}_{2}(12 \mathrm{~mL})$ was added carefully. The resulting suspension was stirred vigorously overnight and then concentrated. The residue was partitioned between water $(50 \mathrm{~mL})$ and $\mathrm{CH}_{2} \mathrm{Cl}_{2}(100 \mathrm{~mL})$. The aqueous layer was extracted with $\mathrm{CH}_{2} \mathrm{Cl}_{2}(75 \mathrm{~mL} \times 2)$. The combined organic layers were washed with water $(60 \mathrm{~mL} \times 3)$ and dried with $\mathrm{Na}_{2} \mathrm{SO}_{4}$. The filtered organic layer was concentrated and the residue was purified by flash chromatography using 15-25\% EA/Hex as the eluting solvent to afford 4 as a clear oil, which solidified just below room temperature. Yield: $3.51 \mathrm{~g}, 86 \% ; R_{f}=0.33$ (25\% EA/Hex); $[\mathrm{a}]_{\mathrm{D}} 19.5$ (c 0.87, $\left.\mathrm{CHCl}_{3}\right) ;{ }^{1} \mathrm{H} \mathrm{NMR}\left(\mathrm{CDCl}_{3}\right) \delta$ $1.15(3 \mathrm{H}, \mathrm{d}, J=7.3 \mathrm{~Hz}), 1.19(3 \mathrm{H}, \mathrm{d}, J=7.0 \mathrm{~Hz}), 1.49-1.69(4 \mathrm{H}$, $\mathrm{m}), 1.95(1 \mathrm{H}, \mathrm{t}, \mathrm{J}=2.6 \mathrm{~Hz}), 2.03-2.25(4 \mathrm{H}, \mathrm{m}), 2.18(2 \mathrm{H}, \mathrm{td}, J=7.1$, $2.6 \mathrm{~Hz}), 2.28(3 \mathrm{H}, \mathrm{s}), 2.43-2.52(1 \mathrm{H}, \mathrm{m}), 2.49(6 \mathrm{H}, \mathrm{s}), 2.55(1 \mathrm{H}, \mathrm{d}$, $J=6.8 \mathrm{~Hz}), 3.61-3.67(1 \mathrm{H}, \mathrm{dtd}, J=3.5,6.8,9.5 \mathrm{~Hz}), 4.11-4.15(1 \mathrm{H}$, $\mathrm{dq}, 4.0$ and $6.9 \mathrm{~Hz}), 4.54\left(1 \mathrm{H}, \mathrm{B}\right.$ of $\left.\mathrm{AB}, J_{\mathrm{AB}}=16.5 \mathrm{~Hz}\right), 4.75(1 \mathrm{H}$, $\mathrm{A}$ of $\left.\mathrm{AB}, J_{\mathrm{AB}}=16.5 \mathrm{~Hz}\right), 4.75(2 \mathrm{H}, \mathrm{s}), 5.86(\mathrm{~d}, 1 \mathrm{H}), 6.87(2 \mathrm{H}, \mathrm{s})$, 6.88-6.90 $(2 \mathrm{H}, \mathrm{m}), 7.17-7.30(8 \mathrm{H}, \mathrm{m}) ;{ }^{13} \mathrm{C} \mathrm{NMR}\left(\mathrm{CDCl}_{3}\right) \delta 174.5$, 148.2, 142.6, 140.3, 138.4, 138.2, 133.4, 132.1, 128.5, 128.4, 128.0, $127.7,127.2,126.0,109.9,84.3,78.3,72.8,68.5,56.8,48.2,45.5$, 35.0, 32.4, 31.8, 26.5, 23.0, 21.0, 18.0, 14.1, 13.5; IR: 3532, 3299, 3031, 2981, 2942, 1737, 1604, 1454, 1381, 1321, 1153, 1013, 858, 757, 699, 659; MS: HR-FAB: calcd for $\mathrm{C}_{38} \mathrm{H}_{47} \mathrm{NO}_{5} \mathrm{~S}$ : $[\mathrm{M}+\mathrm{Li}]^{+}$: 636.3334, found: 636.3346 .

\section{6. (2S,3R)-2-Methyl-6-methyleneundec-10-yne-1,3-diol (4)}

To a stirred solution of the chiral ester 3 (2.6 g, $4.2 \mathrm{mmol})$ in THF $(40 \mathrm{~mL})$ was added lithium aluminum hydride $(0.19 \mathrm{~g}$, $5.0 \mathrm{mmol})$ at $0{ }^{\circ} \mathrm{C}$. The reaction mixture was stirred at room temperature for $1 \mathrm{~h}$ and then quenched by the careful addition of $\mathrm{Na}_{2} \mathrm{SO}_{4} \cdot 10 \mathrm{H}_{2} \mathrm{O}$. The mixture was stirred vigorously for $30 \mathrm{~min}$ and filtered. The residue obtained upon concentration was resuspended in hexane $(60 \mathrm{~mL})$ and then filtered, resulting in recovery of the auxiliary alcohol. The residue obtained upon concentration of the filtrate was separated by chromatography with 1:1 ethyl acetate and hexane to afford the 1,3diol 5 as an clear colorless oil. Yield: $0.64 \mathrm{~g}, 73 \% ; R_{f}=0.27(50 \%$ EA/Hex); [a $]_{\mathrm{D}} 31.7\left(c 1.1, \mathrm{CHCl}_{3}\right) ;{ }^{1} \mathrm{H} \mathrm{NMR}\left(\mathrm{CDCl}_{3}\right) \delta 4.79(1 \mathrm{H}$, s), $4.77(1 \mathrm{H}, \mathrm{s}), 3.77(1 \mathrm{H}, \mathrm{dd}, J=3.7,10.8 \mathrm{~Hz}), 3.55-3.64(2 \mathrm{H}, \mathrm{m})$, $2.90(2 \mathrm{H}$, br s$), 2.04-2.22(6 \mathrm{H}, \mathrm{m}), 1.95(1 \mathrm{H}, \mathrm{t}, J=2.6 \mathrm{~Hz}), 1.53-$ $1.77(5 \mathrm{H}, \mathrm{m}), 0.89(3 \mathrm{H}, \mathrm{d}, \mathrm{J}=7.0 \mathrm{~Hz}) ;{ }^{3} \mathrm{C} \mathrm{NMR}\left(\mathrm{CDCl}_{3}\right) \delta 148.6$, 109.7, 84.3, 76.9, 68.5, 67.5, 39.8, 34.8, 33.2, 31.7, 26.4, 17.9, 13.8; IR: 3302 (br), 2939, 1644, 1432, 1275, 1026; MS: HR-FAB: calcd for $\mathrm{C}_{13} \mathrm{H}_{22} \mathrm{O}_{2}$ : [M+Li] $]^{+}: 217.1780$, found: 217.1778 .

\section{7. (2S,3R)-2-Methyl-6-methylene-1-(triisopropylsilyloxy) undec-10-yn-3-ol (5)}

Diol 4 (0.55 g, $2.6 \mathrm{mmol})$ was dissolved in $\mathrm{CH}_{2} \mathrm{Cl}_{2}(15 \mathrm{~mL})$ and cooled to $0{ }^{\circ} \mathrm{C}$. To this solution were added $\mathrm{Et}_{3} \mathrm{~N}^{2}(0.44 \mathrm{~mL}$, $3.2 \mathrm{mmol}$ ), DMAP (32 mg, $0.26 \mathrm{mmol}$ ), and triisopropylsilyl chloride $(0.61 \mathrm{~mL}, 2.9 \mathrm{mmol})$ sequentially. This mixture was warmed to room temperature and stirred for $15 \mathrm{~h}$, and then quenched with saturated aq $\mathrm{NH}_{4} \mathrm{Cl}$. The aqueous phase was extracted with $\mathrm{Et}_{2} \mathrm{O}$, and the combined organic extracts were dried with $\mathrm{Na}_{2} \mathrm{SO}_{4}$, filtered, and concentrated in vacuo. The resulting crude material was purified by column chromatography using $5 \%$ EA/Hex to afford $0.81 \mathrm{~g}$ of the silyl ether as colorless oil. Yield: $85 \% ; R_{f}=0.44(10 \% \mathrm{EA} / \mathrm{Hex}) ;[\alpha]_{\mathrm{D}} 25.1$ (c 1.2, $\left.\mathrm{CHCl}_{3}\right) ;{ }^{1} \mathrm{H} \mathrm{NMR}\left(\mathrm{CDCl}_{3}\right) \delta 0.85(3 \mathrm{H}, \mathrm{d}, J=7.0 \mathrm{~Hz}), 1.03-1.15$ $(21 \mathrm{H}), 1.49-1.76(5 \mathrm{H}), 1.92(1 \mathrm{H}, \mathrm{t}, J=2.6 \mathrm{~Hz}), 2.02-2.29(6 \mathrm{H}, \mathrm{m})$, $3.54(1 \mathrm{H}, \mathrm{m}), 3.66(1 \mathrm{H}, \mathrm{dd}, J=7.7,9.9 \mathrm{~Hz}), 3.88(1 \mathrm{H}, \mathrm{dd}, J=3.9$, $9.9 \mathrm{~Hz}), 3.97(1 \mathrm{H}, \mathrm{d}, 3.0 \mathrm{~Hz}), 4.72(1 \mathrm{H}, \mathrm{s}), 4.76(1 \mathrm{H}, \mathrm{s}) ;{ }^{13} \mathrm{C} \mathrm{NMR}$ $\left(\mathrm{CDCl}_{3}\right) \delta 148.9,109.2,84.4,76.5,69.1,68.3,39.6,35.1,33.4$, 31.5, 26.5, 17.94, 17.89, 13.7, 11.7; IR: 3439 (br), 3314, 2943, 2886,
1645, 1463, 1386, 1276, 1261, 1062, 996, 883, 797; MS: HR-FAB: calcd for $\mathrm{C}_{22} \mathrm{H}_{42} \mathrm{O}_{2} \mathrm{Si}:[\mathrm{M}+\mathrm{H}]^{+}:$367.3033, found: 367.3042 .

\section{8. (2S,3R)-2-Methyl-6-methylene-1-(triisopropylsilyloxy) undec-10-yn-3-yl methanesulfonate (6)}

Alcohol 5 (0.70 g, $1.9 \mathrm{mmol})$ was dissolved in $\mathrm{CH}_{2} \mathrm{Cl}_{2}$ $(10 \mathrm{~mL})$ and the solution was cooled to $0{ }^{\circ} \mathrm{C}$. $\mathrm{Et}_{3} \mathrm{~N}(0.32 \mathrm{~mL}$, $2.3 \mathrm{mmol})$ and $\mathrm{MsCl}(0.18 \mathrm{~mL}, 2.3 \mathrm{mmol})$ were added and the reaction mixture was allowed to warm to room temperature. After stirring for $14 \mathrm{~h}$, the reaction mixture was diluted with $\mathrm{CH}_{2} \mathrm{Cl}_{2}$ and then washed with satd aq $\mathrm{NH}_{4} \mathrm{Cl}$ and brine and dried with $\mathrm{Na}_{2} \mathrm{SO}_{4}$. The crude mesylate obtained upon concentration was purified by chromatography $(10 \% \mathrm{EA} / \mathrm{Hex})$ to afford $0.69 \mathrm{~g}$ of mesylate 6 . Yield: $0.69 \mathrm{~g}, 81 \% ; R_{f}=0.26(10 \%$ EA/Hex); [a $]_{\mathrm{D}} 7.0\left(\right.$ c 0.97, $\left.\mathrm{CHCl}_{3}\right) ;{ }^{1} \mathrm{H} \mathrm{NMR}\left(\mathrm{CDCl}_{3}\right) \delta 0.97(3 \mathrm{H}$, $\mathrm{d}, J=7.0 \mathrm{~Hz}), 1.04-1.11(21 \mathrm{H}), 1.62-1.1 .70(2 \mathrm{H}), 1.77-1.89(2 \mathrm{H})$, $1.94(1 \mathrm{H}, \mathrm{t}, J=2.6 \mathrm{~Hz}), 2.03-2.27(7 \mathrm{H}), 3.00(3 \mathrm{H}, \mathrm{s}), 3.63(1 \mathrm{H}, \mathrm{s})$, $3.65(1 \mathrm{H}, \mathrm{s}), 4.78(2 \mathrm{H}, \mathrm{s}), 4.89(1 \mathrm{H}, \mathrm{dt}, J=3.9,8.8 \mathrm{~Hz}) ;{ }^{13} \mathrm{C}$ NMR $\left(\mathrm{CDCl}_{3}\right) \delta 147.5,110.1,85.1,84.2,68.5,65.0,39.7,38.6,34.9,31.6$, 28.4, 26.5, 18.0, 17.7, 11.9, 11.6; IR: 3312, 2943, 2893, 2866, 1646, 1463, 1336, 1175, 1109, 908, 884, 795, 678; MS: HR-FAB: calcd for $\mathrm{C}_{23} \mathrm{H}_{44} \mathrm{O}_{4} \mathrm{SSi}:[\mathrm{M}+\mathrm{H}]^{+}$: 445.2809, found: 445.2805.

\subsection{6-Hydroperoxy-6-methoxy-2-methyl- 1-(triisopropylsilyloxy) undec-10-yn-3-ylmethanesulfonate (7a and $7 b$ )}

A stream of $\mathrm{O}_{3} / \mathrm{O}_{2}$ was bubbled into a $-78{ }^{\circ} \mathrm{C}$ solution of mesylate $7(0.82 \mathrm{~g}, 1.8 \mathrm{mmol})$ in $15 \mathrm{~mL}$ methanol and $50 \mathrm{~mL}$ $\mathrm{CH}_{2} \mathrm{Cl}_{2}$ until the starting material had disappeared (TLC). The reaction was sparged with $\mathrm{N}_{2}$ and then allowed to warm to room temperature and concentrated in vacuo. The residue was purified through a short flash column using 30\% EA/ Hex to afford $0.77 \mathrm{~g}(88 \%)$ of a 1:1 mixture of diastereomers 7a and $7 \mathrm{~b}$. Yield: $0.77 \mathrm{~g}, 88 \% ; R_{f}=0.27$ (33\% EA/Hex); ${ }^{13} \mathrm{C}$ NMR $\left(\mathrm{CDCl}_{3}\right) \delta 108.2,108.0,84.8,84.3,83.8,83.7,77.2,77.3,69.0,68.9$, $48.7,48.6,39.6,39.2,38.7,38.5,29.6,29.0,27.0,24.8,24.6,22.61$, 22.60, 18.41, 18.40, 18.0, 12.1, 11.9, 11.8; MS: HRFAB: calcd for $\mathrm{C}_{23} \mathrm{H}_{46} \mathrm{O}_{7} \mathrm{SSi}:\left[\mathrm{M}-\mathrm{HO}_{2}\right]^{+}:$461.2675, found: 461.2765 .

Small quantities of pure diastereomers could be separated by analytical HPLC (EA/Hex). ${ }^{22}$ Compound 7a $(2 S, 3 R, 6 R)$; ${ }^{1} \mathrm{H}$ NMR $\left(\mathrm{CDCl}_{3}\right) \delta 7.61(1 \mathrm{H}, \mathrm{s}), 4.87-4.91(1 \mathrm{H}, \mathrm{m}), 3.68(1 \mathrm{H}$, s), $3.66(1 \mathrm{H}, \mathrm{s}), 3.32(3 \mathrm{H}, \mathrm{s}), 3.04(3 \mathrm{H}, \mathrm{s}), 2.18-2.28(3 \mathrm{H}, \mathrm{m}$ (contains $2 \mathrm{H}, \mathrm{dt}, J=2.6,6.7 \mathrm{~Hz}$ at $2.27 \mathrm{ppm})), 1.99(1 \mathrm{H}, \mathrm{t}, J=2.6 \mathrm{~Hz})$, $1.90-1.98(1 \mathrm{H}, \mathrm{m}), 1.72-1.82(5 \mathrm{H}, \mathrm{m}), 1.50-1.63(3 \mathrm{H}, \mathrm{m}), 1.03-$ $1.14(21 \mathrm{H}, \mathrm{m}), 0.98(3 \mathrm{H}, \mathrm{d}, J=7.0 \mathrm{~Hz})$. Compound $7 \mathrm{~b}(2 \mathrm{~S}, 3 \mathrm{R}, 6 \mathrm{~S})$; ${ }^{1} \mathrm{H}$ NMR $\left(\mathrm{CDCl}_{3}\right) \delta 8.08(1 \mathrm{H}, \mathrm{s}), 4.91-4.95(1 \mathrm{H}, \mathrm{m}), 3.69(1 \mathrm{H}, \mathrm{s})$, $3.68(1 \mathrm{H}, \mathrm{s}), 3.34(3 \mathrm{H}, \mathrm{s}), 3.05(3 \mathrm{H}, \mathrm{s}), 2.14-2.28(3 \mathrm{H}, \mathrm{m}$ (contains $2 \mathrm{H}, \mathrm{dt}, J=2.6,6.7 \mathrm{~Hz}$ at $2.26 \mathrm{ppm})), 1.88-2.0(3 \mathrm{H}, \mathrm{m}$ (contains $1 \mathrm{H}, \mathrm{t}, J=2.6 \mathrm{~Hz})), 1.66-1.80(4 \mathrm{H}, \mathrm{m}), 1.50-1.63(3 \mathrm{H}, \mathrm{m})$, 1.03-1.14 (21H, m), $0.98(3 \mathrm{H}, \mathrm{d}, J=7.0 \mathrm{~Hz})$.

\subsection{0. (2S)-1-Triisopropylsiloxy-2-((3S,6R)-6-methoxy-6-(pent- 4-ynyl)-1,2-dioxan-3-yl) propane (8a)}

To a solution of hydroperoxyacetal (7a) (17.3 mg, $0.03 \mathrm{mmol})$ in anhydrous toluene $(0.4 \mathrm{~mL})$ was added 18-crown-6 (9.5 mg, $0.04 \mathrm{mmol})$, followed by KOt-Bu (5.8 mg, $0.05 \mathrm{mmol})$. The reaction mixture was stirred for $20 \mathrm{~min}$, at which point additional $\mathrm{KO} t-\mathrm{Bu}(3.3 \mathrm{mg}, 0.03 \mathrm{mmol})$ was added. After stirring for an additional $20 \mathrm{~min}$, the reaction became yellow, and TLC indicated the absence of starting material. The crude reaction mixture was filtered through a short pad of silica gel with $20 \%$ EA/Hex. The filtrate was concentrated and the residue was purified by column chromatography using 5\% EA/Hex to afford dioxane 8a. Yield: $11.3 \mathrm{mg}$, 
$79 \% ; R_{f}=0.36(5 \% \mathrm{EA} / \mathrm{Hex}) ;[\mathrm{a}]_{\mathrm{D}}-144\left(\mathrm{c} 0.74, \mathrm{CHCl}_{3}\right) ;{ }^{1} \mathrm{H} \mathrm{NMR}$ $\left(\mathrm{CDCl}_{3}\right) \delta 4.05(1 \mathrm{H}, \mathrm{ddd}, J=1.9,6.3,11.0 \mathrm{~Hz}), 3.68(1 \mathrm{H}, \mathrm{dd}$, $J=5.2,9.8 \mathrm{~Hz}), 3.63(1 \mathrm{H}, \mathrm{dd}, J=5.6,9.8 \mathrm{~Hz}), 3.28(3 \mathrm{H}, \mathrm{s}), 2.19-$ $2.24(2 \mathrm{H}, \mathrm{m}), 1.96(1 \mathrm{H}, \mathrm{t}, \mathrm{J}=2.6 \mathrm{~Hz}), 1.41-1.92(9 \mathrm{H}, \mathrm{m}), 1.03-1.13$ $(21 \mathrm{H}, \mathrm{m}), 1.01(3 \mathrm{H}, \mathrm{d}, J=6.9 \mathrm{~Hz}) ;{ }^{13} \mathrm{C} \mathrm{NMR}\left(\mathrm{CDCl}_{3}\right) \delta 102.3,83.8$, 81.7, 68.8, 65.1, 48.4, 39.7, 31.9, 30.7, 22.9, 22.1, 18.5, 18.0, 12.7, 12.0; IR: 3312, 2943, 2866, 1462, 1238, 1109, 1066, 917, 882, 789, 746, 682; MS: HR-FAB: calcd for $\mathrm{C}_{22} \mathrm{H}_{42} \mathrm{O}_{4} \mathrm{Si}$ : $[\mathrm{M}+\mathrm{Li}]^{+}$: 405.3012, found: 405.3000 .

\subsection{1. (2S)-1-Triisopropylsiloxy-2-((3S,6S)-6-methoxy-6-(pent- 4-ynyl)-1,2-dioxan-3-yl) propane (8b)}

By a similar procedure, hydroperoxyacetal (7b) (20 mg, $0.04 \mathrm{mmol}$ ) was converted to 1,2-dioxane $8 \mathrm{~b}$. Yield: $14.5 \mathrm{mg}$, $87 \% ; R_{f}=0.36$ (5\% EA/Hex); $[\mathrm{a}]_{\mathrm{D}}+121.4$ (c 1.4, $\left.\mathrm{CHCl}_{3}\right) ;{ }^{1} \mathrm{H} \mathrm{NMR}$ $\left(\mathrm{CDCl}_{3}\right) \delta 3.93(1 \mathrm{H}, \mathrm{dt}, J=8.5,5.7 \mathrm{~Hz}), 3.70(1 \mathrm{H}, \mathrm{dd}, J=4.7,9.9 \mathrm{~Hz})$, $3.67(1 \mathrm{H}, \mathrm{dd}, J=4.7,8.8 \mathrm{~Hz}), 3.34(3 \mathrm{H}, \mathrm{s}), 2.25(2 \mathrm{H}, \mathrm{dt}, J=2.6$ and $6.7 \mathrm{~Hz}), 2.03-2.15(2 \mathrm{H}, \mathrm{m}), 1.99(1 \mathrm{H}, \mathrm{t}, J=2.6 \mathrm{~Hz}), 1.75-1.86(2 \mathrm{H}$, $\mathrm{m}), 1.54-1.74(5 \mathrm{H}, \mathrm{m}), 1.12(3 \mathrm{H}, \mathrm{d}, J=6.8 \mathrm{~Hz}), 1.04-1.10(21 \mathrm{H}, \mathrm{m})$; ${ }^{13} \mathrm{C} \mathrm{NMR}\left(\mathrm{CDCl}_{3}\right) \delta 102.3,83.8,81.7,68.8,65.1,48.4,39.7,31.9$, 30.7, 22.9, 22.1, 18.5, 18.0, 12.7, 12.0; IR: 3312, 2943, 2866, 1462, 1238, 1109, 1066, 917, 882, 789, 746, 682; MS: HR-FAB: calcd for $\mathrm{C}_{22} \mathrm{H}_{42} \mathrm{O}_{4} \mathrm{Si}:[\mathrm{M}+\mathrm{Li}]^{+}$: 405.3012, found: 405.3000 .

\subsection{One pot ozonolysis/cyclization method}

\subsection{1. (2S)-1-Triisopropylsiloxy-2-((3S,6RS)-6-methoxy-6-(pent-} 4-ynyl)-1,2-dioxan-3-yl)propane (8ab)

Mesylate $6(0.6811 \mathrm{~g}, 1.5 \mathrm{mmol})$ was dissolved in $20 \mathrm{~mL}$ $\mathrm{CH}_{2} \mathrm{Cl}_{2}$ and methanol $(7.8 \mathrm{~mL}, 193 \mathrm{mmol})$. The solution was cooled to $-78{ }^{\circ} \mathrm{C}$ and treated with a stream of $\mathrm{O}_{3} / \mathrm{O}_{2}$. The reaction was monitored by TLC until starting material was absent. The reaction mixture was then sparged with nitrogen and allowed to warm to room temperature. To this solution was added 18-crown-6 (396.8 mg, $1.5 \mathrm{mmol})$, followed by KOt$\mathrm{Bu}(154.2 \mathrm{mg}, 1.4 \mathrm{mmol})$. The reaction mixture was stirred for $20 \mathrm{~min}$, at which point additional $\mathrm{KO} t-\mathrm{Bu}(170.5 \mathrm{mg}, 1.5 \mathrm{mmol})$ was added. After stirring for an additional $20 \mathrm{~min}$, the reaction became yellow, and TLC indicated the absence of starting material. The crude reaction mixture was filtered through a short pad of silica gel with $20 \% \mathrm{EA} / \mathrm{Hex}$. The filtrate was concentrated and the residue was purified by column chromatography using 5\% EA/Hex to afford $458.4 \mathrm{mg}(75 \%)$ of an approximately 1:1 mixture of $\mathbf{8 a}$ and $\mathbf{8 b}$. Yield: $75 \%$.

\subsection{3. (2S)-1-Triisopropylsiloxy-2-((3S,6R)-6-methoxy-6-((4E)- 4-iodopent-4-enyl)-1,2-dioxan-3-yl) propane (9)}

1,2-Dioxane 8a (0.43 g, $1.1 \mathrm{mmol})$, azeotropically dried by concentration from distilled toluene $(2 \times 15 \mathrm{~mL})$, was dissolved in $10 \mathrm{~mL}$ dry toluene. To this solution was added $\mathrm{Cp}_{2} \mathrm{ZrHCl}$ $(0.38 \mathrm{~g}, 1.5 \mathrm{mmol})$ under a nitrogen atmosphere. After stirring for $15 \mathrm{~min}$, the solution became light orange in color. Another portion of $\mathrm{Cp}_{2} \mathrm{ZrHCl}(0.21 \mathrm{~g}, 0.8 \mathrm{mmol})$ was added and the reaction mixture was stirred for another $15 \mathrm{~min}$. Iodine $(0.41 \mathrm{~g}$, $1.6 \mathrm{mmol}$ ) was added slowly until the dark red color became permanent. The reaction mixture was diluted with $50 \mathrm{~mL} \mathrm{Hex}$, and filtered through Celite. The filtrate was washed with saturated aqueous $\mathrm{Na}_{2} \mathrm{~S}_{2} \mathrm{O}_{3}$, dried with $\mathrm{Na}_{2} \mathrm{SO}_{4}$, and concentrated. The residue was purified by column chromatography using $5-10 \%$ EA/Hex to afford $0.43 \mathrm{~g}(76 \%)$ of a $1: 3$ mixture of the terminal alkene reduction product (due to water) and the desired terminal E-iodoalkene 9, which could be separated by semi-prep HPLC (EA/Hex); $R_{f}=0.32\left(5 \%\right.$ EA/Hex); $[\alpha]_{\mathrm{D}}-97.2$ (c $\left.0.94, \mathrm{CHCl}_{3}\right) ;{ }^{1} \mathrm{H} \mathrm{NMR}\left(\mathrm{CDCl}_{3}\right) \delta 6.48(1 \mathrm{H}, \mathrm{dt}, J=14.4,7.1 \mathrm{~Hz})$,
$6.01(1 \mathrm{H}, \mathrm{dt}, J=14.4,1.3 \mathrm{~Hz}), 4.01-4.06(1 \mathrm{H}, \mathrm{m}), 3.67(1 \mathrm{H}, \mathrm{dd}$ $J=5.2,9.9 \mathrm{~Hz}), 3.63(1 \mathrm{H}, \mathrm{dd}, J=5.6,9.9 \mathrm{~Hz}), 3.24(3 \mathrm{H}, \mathrm{s}), 2.03-$ $2.08(2 \mathrm{H}, \mathrm{m}), 1.82-1.92(2 \mathrm{H}, \mathrm{m}), 1.24-1.67(7 \mathrm{H}, \mathrm{m}), 1.03-1.12$ $(21 \mathrm{H}, \mathrm{m}), 1.00(3 \mathrm{H}, \mathrm{d}, J=6.9 \mathrm{~Hz}) ;{ }^{13} \mathrm{C} \mathrm{NMR}\left(\mathrm{CDCl}_{3}\right) \delta 145.7$ 102.3, 81.7, 75.2, 65.0, 48.4, 39.6, 36.0, 32.1, 30.7, 22.9, 21.8, 18.0, 12.7, 11.9; IR: 2942, 2865, 1462, 1234, 1110, 1070, 1030, 946, 882, 788, 683, 659; MS: HR-FAB: calcd for $\mathrm{C}_{22} \mathrm{H}_{43} \mathrm{LiO}_{4} \mathrm{Si}$ : $[\mathrm{M}+\mathrm{Li}]^{+}$: 533.2136, found: 533.2125 .

\subsection{4. (2S)-1-Triisopropylsiloxy-2-((3S,6R)-6-methoxy- 6-((4E,6E)-nona-4,6-dienyl)-1,2-dioxan-3-yl) propane (10)}

1-Butyne (0.12 g, $2.2 \mathrm{mmol})$ was condensed into a $50 \mathrm{~mL}$ flask at $-78{ }^{\circ} \mathrm{C}$ under a $\mathrm{N}_{2}$ atmosphere, and dissolved in distilled THF $(4 \mathrm{~mL})$ and distilled toluene $(4 \mathrm{~mL})$. The solution was warmed slowly to $0{ }^{\circ} \mathrm{C}$, whereupon $\mathrm{Cp}_{2} \mathrm{ZrHCl}(0.22 \mathrm{~g}$, $0.85 \mathrm{mmol}$ ) was added. The reaction was held at $0{ }^{\circ} \mathrm{C}$ for $1 \mathrm{~h}$, after which another portion of $\mathrm{Cp}_{2} \mathrm{ZrHCl}(0.10 \mathrm{~g}, 0.39 \mathrm{mmol})$ was added. The reaction mixture was stirred for another hour at $0{ }^{\circ} \mathrm{C}$ then the ice bath was removed. After $10 \mathrm{~min}$, the solution was cooled to $0{ }^{\circ} \mathrm{C}$ again and $\mathrm{ZnCl}_{2}(0.12 \mathrm{~g}, 0.88 \mathrm{mmol})$ in $2 \mathrm{~mL}$ dry THF was added. ${ }^{25}$ The reaction solution turned orange and was stirred at $0{ }^{\circ} \mathrm{C}$ for $10 \mathrm{~min}$. Vinyl iodide 9 $(0.16 \mathrm{~g}, 0.30 \mathrm{mmol})$ was added, followed by $\mathrm{Pd}\left(\mathrm{PPh}_{3}\right)_{4}(45 \mathrm{mg}$, $0.04 \mathrm{mmol}$ ). The reaction was stirred at $0{ }^{\circ} \mathrm{C}$ for $5 \mathrm{~min}$. TLC indicated the presence of significant amount of starting material, and another portion of $\mathrm{Pd}\left(\mathrm{PPh}_{3}\right)_{4}(15 \mathrm{mg}, 0.01 \mathrm{mmol})$ was added. The reaction mixture was stirred at $0{ }^{\circ} \mathrm{C}$ for an additional $0.5 \mathrm{~h}$, at which point TLC indicated the absence of starting material. The reaction was diluted with $20 \mathrm{~mL}$ hexane, and the reaction mixture was filtered through a short pad of silica gel with $30 \%$ EA/Hex. The filtrate was concentrated in vacuo and the residue was purified by flash chromatography ( $5 \% \mathrm{EA} / \mathrm{Hex}$ ) to give $0.13 \mathrm{~g}$ of an inseparable mixture of diene $\mathbf{1 0}$ and terminal alkene 11. The coupled and reduced products were separable after removal of the TIPS ether (next step).

\subsection{5. (2S)-2-((3S,6R)-6-Methoxy-6-((4E,6E)-nona-4,6-dienyl)- 1,2-dioxan-3-yl) propan-1-ol (12)}

A few milligrams of the mixture of diene and terminal alkene from the previous step was dissolved in THF. A solution of TBAF (nominally $1 \mathrm{M}$ in THF) was added dropwise until TLC indicated the disappearance of the starting material. The reaction mixture was washed with saturated ammonium chloride solution and water sequentially. Then the separated organic layer was dried with $\mathrm{Na}_{2} \mathrm{SO}_{4}$, concentrated. The residue was first purified by flash chromatography, and then by HPLC. The desired deprotected diene product was very easily isolated by $\mathrm{HPLC} ;{ }^{1} \mathrm{H} \mathrm{NMR}\left(\mathrm{CDCl}_{3}\right) \delta 5.95-6.06(2 \mathrm{H}, \mathrm{m})$, 5.49-5.66 (2H, m), 4.10-4.14 (1H, m), 3.54-3.70 (m, $2 \mathrm{H}), 3.25$ $(3 \mathrm{H}, \mathrm{s}), 2.04-2.20(4 \mathrm{H}, \mathrm{m}), 1.82-2.01(3 \mathrm{H}, \mathrm{m}), 1.25-1.72(6 \mathrm{H}, \mathrm{m})$, $0.99(3 \mathrm{H}, \mathrm{t}, J=7.4 \mathrm{~Hz}), 0.96(3 \mathrm{H}, \mathrm{d}, J=7.2 \mathrm{~Hz}) ;{ }^{13} \mathrm{C}$ NMR $\delta$ 134.5, 131.2, 131.0, 129.1, 102.8, 82.4, 65.1, 48.4, 39.1, 32.5, 32.2, 30.5, 25.6, 22.7, 21,6, 13.6, 11.9; MS: HRFAB: calcd for $\mathrm{C}_{17} \mathrm{H}_{30} \mathrm{O}_{4} \mathrm{Li}$ : $[\mathrm{M}+\mathrm{Li}]^{+}$: 305.2304, found: 305.2305 (0.2 ppm).

\section{Acknowledgments}

Research was conducted in facilities remodeled with support from NIH (RR016544-01). NMR spectra were acquired, in part, on spectrometers purchased with NSF support (MRI 0079750 and CHE 0091975). We thank Dr. Don Davies and Ms. Laure Chauset for some initial experiments related to alkylation of hydroperoxyacetals, and Ms. Carita Kordik for assistance with mass spectrometry. 


\section{References and notes}

1 D. A. Casteel, Nat. Prod. Rep. 16 (1999), p. 55.

2 M. Kobayashi, K. Kondo, and I. Kitagawa, Chem. Pharm. Bull. 41 (1993), p. 1324.

3 (a) N. Murakami, M. Kawanishi, S. Itagaki, T. Horii, and M. Kobayashi, Tetrahedron Lett. 42 (2001), p. 7281. (b) M. Kawanishi, N. Kotoku, S. Itagaki, T. Horii, and M. Kobayashi, Bioorg. Med. Chem. 12 (2004), p. 5297 and references within.

4 For recent applications of conjugate addition for the conjugate addition of hydroperoxyacetals to enoates, see: H.X. Jin, Q. Zhang, H.-S. Kim, Y. Wataya, H.-H. Liu, and Y. Wu, Tetrahedron 62 (2006), p. 7699. H.-H. Liu, H.-X. Jin, Q. Zhang, Y.-K. Wu, H.-S. Kim, and Y. Wataya, Chin. J. Chem. 23 (2005), p. 1469.

5 For leading references to synthesis of some other classes of six-membered ring peroxide natural products, see: $\mathrm{M}$. Jung, J. Ham, and J. Song, Org. Lett. 4 (2002), p. 2763. G. Yao and K. Steliou, Org. Lett. 4 (2002), p. 485. B. B. Snider and Z. Shi, J. Am. Chem. Soc. 114 (1992), p. 1790. P. H. Dussault, C. T. Eary, and K. R. Woller, J. Org. Chem. 64 (1999), p. 1789.

6 For the asymmetric synthesis of an alkoxydioxane via chemoselective hydrogenation of a 1,2-dioxine, see: P. H. Dussault, S. Kreifels, and I. Q. Lee, Synth. Commun. 25 (1995), p. 2613.

7 D. A. Casteel, Nat. Prod. Rep. 9 (1992), p. 289.

8 Portions of this work have been reported in C. Xu, Total Synthesis of Peroxyacarnoic Acids and Peroxyplakoric Acids. New Synthetic Methodology for Organic Peroxides and Application of Peroxides as Enzyme Inhibitors, University of NebraskaLincoln: Lincoln, NE, (2006) ISBN:9780542654350.

9 For leading references to synthesis of peroxide heterocycles, see E. Korshin and M. D. Bachi in Z. Rappoport, ed., Chemistry of Peroxides vol. 2, John Wiley \& Sons, Chichester (2006), pp. 189-305.

10 For leading references to radical promoted synthesis of peroxide heterocycles, see H. Nishino, Top. Heterocycl. Chem. 6 (2006), p. 39.

11 For leading references to electrophilic cyclizations, see Reference 9 and J. R. Harris, S. R. Waetzig, and K. A. Woer- pel, Org. Lett. 11 (2009), p. 3290. Y. Ushigoe, Y. Kano, and M. Nojima, J. Chem. Soc., Perkin Trans. 1 (1997), p. 5. P. H. Dussault and D. R. Davies, Tetrahedron Lett. 37 (1996), p. 463.

12 P. A. Bartlett and C. Chapuis, J. Org. Chem. 51 (1986), p. 2799.

13 Previous approaches to peroxyplakoric analogs have focused on targets lacking the exocyclic stereocenter: see References $3 \mathrm{~b}$ and 4 .

14 C. Xu, J. M. Raible, and P. H. Dussault, Org. Lett. 7 (2005), p. 2509.

15 X. Lu, Y. Liu, B. Sun, B. Cindric, and L. Deng, J. Am. Chem. Soc. 130 (2008), p. 8134.

16 X.-X. Xu and H.-Q. Dong, J. Org. Chem. 60 (1995), p. 3039.

17 P. Dai and P. H. Dussault, Org. Lett. 7 (2005), p. 4333.

18 P. H. Dussault and U. R. Zope, J. Org. Chem. 60 (1995), p. 8218.

19 P. Ghorai, P. H. Dussault, and C. Hu, Org. Lett. 10 (2008), p. 2401.

20 P. H. Dussault, C. T. Eary, R. J. Lee, and U. R. Zope, J. Chem. Soc., Perkin Trans. 1 (1999), p. 2189.

21 T. Inoue, J.-F. Liu, D. C. Buske, and A. Abiko, J. Org. Chem. 67 (2002), p. 5250. A. Abiko, Acc. Chem. Res. 37 (2004), p. 387. A. Abiko, Org. Synth. 79 (2003), p. 116.

22 Hydroperoxyketals $7 \mathbf{a}$ and $7 \mathbf{b}$ are relatively labile. While we were sometimes able to separate small amounts of individual epimers, extensive chromatography more often resulted in partial decomposition to form the corresponding ketone.

23 See, for example S. Sperry, F. A. Valeriote, T. H. Corbett, and P. Crews, J. Nat. Prod. 61 (1998), p. 241.

24 P. H. Dussault and C. T. Eary, J. Am. Chem. Soc. 120 (1998), p. 7133.

25 P. Wipf and R. L. Nunes, Tetrahedron 60 (2004), p. 1269.

26 Plakortin: M. D. Higgs, and D. J. Faulkner, J. Org. Chem. 43 (1978), p. 3454. F. Cafieri, E. Fattorusso, O. Taglialatela-Scafati and A. Ianaro, Tetrahedron 55 (1999), p. 7045.

27 Plakortolide E: M. Varoglu, B. M. Peters, and P. Crews, J. Nat. Prod. 58 (1995), p. 27.

28 L. Smith and F. L. Hill, J. Chromatogr. 66 (1972), p. 101. 